\title{
Symmetric Equations for Evaluating Maximum Torsion Stress of Rectangular Beams in Compliant Mechanisms
}

\author{
Gui-Min Chen ${ }^{1,2}$ and Larry L. Howell ${ }^{2^{*}}$ (1)
}

\begin{abstract}
There are several design equations available for calculating the torsional compliance and the maximum torsion stress of a rectangular cross-section beam, but most depend on the relative magnitude of the two dimensions of the crosssection (i.e., the thickness and the width). After reviewing the available equations, two thickness-to-width ratio independent equations that are symmetric with respect to the two dimensions are obtained for evaluating the maximum torsion stress of rectangular cross-section beams. Based on the resulting equations, outside lamina emergent torsional joints are analyzed and some useful design insights are obtained. These equations, together with the previous work on symmetric equations for calculating torsional compliance, provide a convenient and effective way for designing and optimizing torsional beams in compliant mechanisms.
\end{abstract}

Keywords: Compliant mechanism, Maximum torsion stress, Rectangular beam, Lamina emergent joint

\section{Introduction}

A compliant mechanism achieves its mobility through the deflections of its compliant elements [1]. In most compliant mechanisms, the compliant elements are designed to produce motion through bending deflections [2-4]. In fact, torsional deflections could be another valuable source for obtaining mobility in compliant mechanisms. There have been successful designs utilizing torsional deflections, for example, a split-tube flexure based on the torsion of an open-section hollow shaft was presented [5], a revolute joint comprised of two crossed torsion plates which shows a good performance in resisting axis drift was presented [6], torsional micromirrors were proposed for optical switches and optical displays $[7,8]$, a torsional micro-resonator was fabricated for mass sensing [9], lamina emergent torsional (LET) joints were devised to facilitate the design of compliant mechanisms that can be fabricated from a planar material but have motion that emerges out of the fabrication plane [10-12]

\footnotetext{
*Correspondence: Ihowell@byu.edu

${ }^{2}$ Department of Mechanical Engineering, Brigham Young University, Provo, UT 84602, USA

Full list of author information is available at the end of the article
}

and were employed in precision adjustment mechanisms [13], torsion hinges were proposed as surrogate folds in origami-based engineering design $[14,15]$, and torsional beams were successfully used for achieving static balancing of an inverted pendulum [16].

There are several design equations available for calculating the torsional compliance and maximum torsion stress of a rectangular cross-section beam. However, before using these equations, one of the two dimensions (i.e., the thickness and the width) of the cross-section must be defined as the wider of the two dimensions [17]. This situation might be troublesome and error-prone during the design phase because we always do not know which dimension is larger in advance. This is especially true for an optimization design process considering that the two dimensions of the torsion beam(s) may change greatly during the design iteration process including the relative size of the two dimensions. In our previous work [17], general compliance equations that are symmetric with respect to the two dimensions were obtained to facilitate the design of torsional beams in compliant mechanisms. These equations had been used in characterizing parasitic motions of compliant mechanisms [18,
SpringerOpen

(c) The Author(s) 2018. This article is distributed under the terms of the Creative Commons Attribution 4.0 International License (http://creativecommons.org/licenses/by/4.0/), which permits unrestricted use, distribution, and reproduction in any medium, provided you give appropriate credit to the original author(s) and the source, provide a link to the Creative Commons license, and indicate if changes were made. 
19] and spatial deflections modeling [20-22]. However, there still lacks an equation for predicting the maximum stress in torsional beams that is symmetric with respect to the two dimensions.

To complement previous work [17], this paper is going to address this absence. The organization of this paper is as follows: Section 2 presents a brief summary on various equations for predicting the maximum stress in torsional beams; two maximum stress equations that are symmetric with respect to the two dimensions of the cross-section are formulated in Section 3; Section 4 offers some design insights for outside lamina emergent joints using the proposed equations; and Section 5 has concluding remarks.

\section{Various Equations for Calculating Maximum Shearing Stress}

For torsion of rectangular sections, the stress at each corner is zero, and the maximum shearing stress $\tau_{\max }$ occurs at the middle points of the longer sides (the points most remote from the centroid of the cross section), as illustrated in Figure 1. It can be obtained using the membrane analogy by assuming $w \geq t[23]$ :

$$
\tau_{\max }=G \theta t\left[1-\frac{8}{\pi^{2}} \sum_{n=1,3,5 \ldots}^{\infty} \frac{1}{n^{2} \cosh (n \pi w / 2 t)}\right],
$$

where $\theta$ is the angle of twist per unit length. The infinite series on the right side converges rapidly. Because the torque as a function of $\theta$ is given as [23]

$$
T=\frac{G \theta w t^{3}}{3}\left[1-\frac{192 t}{\pi^{5} w} \sum_{n=1,3,5 \ldots}^{\infty} \frac{\tanh (n \pi w / 2 t)}{n^{5}}\right] .
$$

Dividing Eq. (1) by Eq. (2) yields the maximum shearing stress as a function of the torque in the form

$$
\tau_{\max }=\frac{3 T}{w t^{2}} Q,
$$

where $Q$ (denoted as $Q_{s}$ for this infinite series expression) is a constant whose value depends only on ratio $t / w(t / w$ $\leq 1)$ :

$$
Q_{s}=\frac{1-\frac{8}{\pi^{2}} \sum_{n=1,3,5 \ldots}^{\infty} \frac{1}{n^{2} \cosh (n \pi w / 2 t)}}{1-\frac{192}{\pi^{5}} \frac{t}{w} \sum_{n=1,3,5 \ldots}^{\infty} \frac{\tanh (n \pi w / 2 t)}{n^{5}}} .
$$

For narrow rectangular sections $(t / w<0.1), Q$ approximately equals 1 .

A polynomial fit of $Q_{s}$ as a function of $t / w$ given in Eq. (4) [24]:

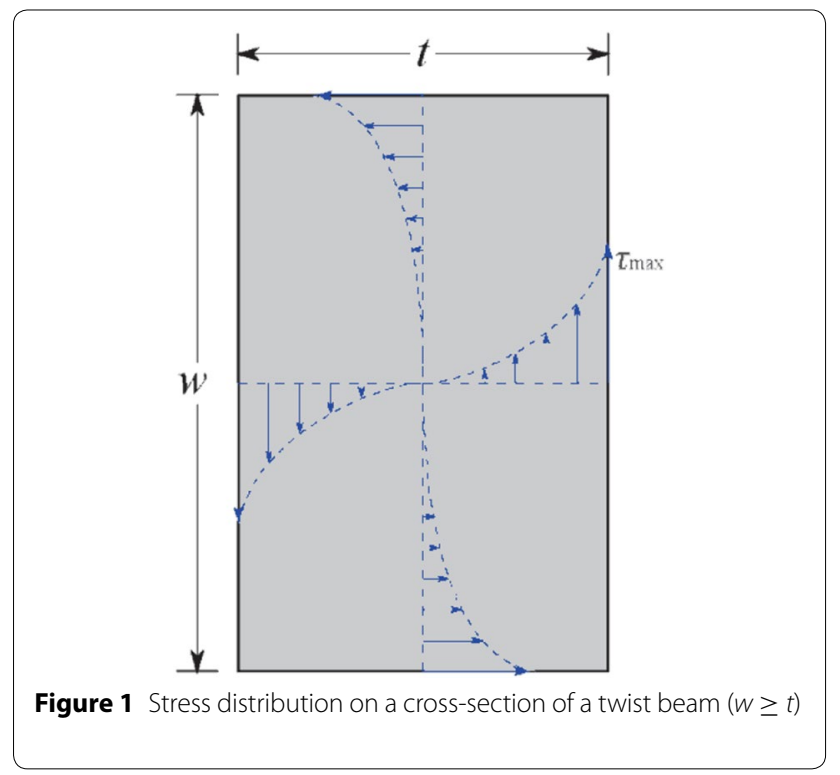

$Q_{\mathrm{r}}=1+0.6095 \frac{t}{w}+0.8865 \frac{t^{2}}{w^{2}}-1.8023 \frac{t^{3}}{w^{3}}+0.91 \frac{t^{4}}{w^{4}}$.

Substituting into Eq. (3) results in the following expres$\operatorname{sion}$ for $\tau_{\max }$ :

$$
\begin{aligned}
\tau_{\max }= & \frac{3 T}{w t^{2}}\left(1+0.6095 \frac{t}{w}+0.8865 \frac{t^{2}}{w^{2}}\right. \\
& \left.-1.8023 \frac{t^{3}}{w^{3}}+0.91 \frac{t^{4}}{w^{4}}\right) .
\end{aligned}
$$

Pilkey [25] approximated $1 / Q$ using the following polynomial expression:

$$
\frac{1}{Q_{\mathrm{p}}}=1-0.63 \frac{t}{w}+0.25 \frac{t^{2}}{w^{2}},
$$

which leads to another expression for $\tau_{\max }$ :

$$
\tau_{\max }=\frac{3 T}{w t^{2}} \frac{1}{\left(1-0.63 \frac{t}{w}+0.25 \frac{t^{2}}{w^{2}}\right)} .
$$

The following expression employing a linear approximation of $Q$ [26] is often used by researchers [10]:

$$
Q_{l}=1+0.6 \frac{t}{w},
$$

and we have

$$
\tau_{\max }=\frac{T}{w^{2} t^{2}}(3 w+1.8 t)=\frac{3 T}{w t^{2}}\left(1+0.6 \frac{t}{w}\right),
$$

which approximately equals the first two terms of Eq. (6). 


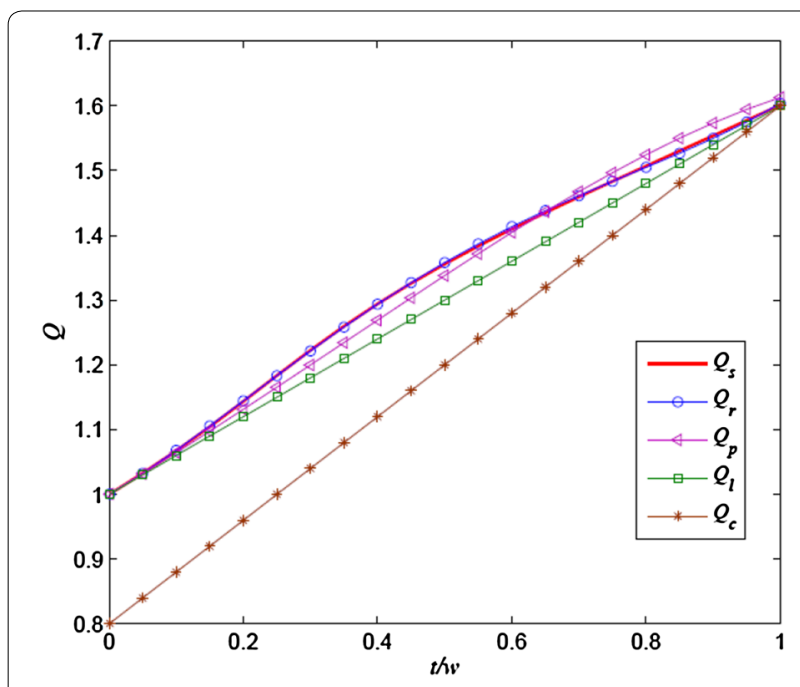

Figure 2 Curves of different expressions for $Q$

Figure 2 compares $Q, Q_{r}, Q_{p}$ and $Q_{l}$. By denoting the errors as:

$$
\begin{gathered}
E_{r}=\frac{Q_{r}-Q_{s}}{Q_{s}}, \\
E_{p}=\frac{Q_{p}-Q_{s}}{Q_{s}}, \\
E_{l}=\frac{Q_{l}-Q_{s}}{Q_{s}} .
\end{gathered}
$$

Figure 3 shows that $Q_{r}$ has an maximum error less than $0.3 \%$, the maximum error of $Q_{p}$ is less than $2 \%$, while $Q_{l}$ may result in an error up to $4.5 \%$.

When using the expressions for $\tau_{\max }$ given in Eqs. (1), (6), (8) and (10), one of the two dimensions of the

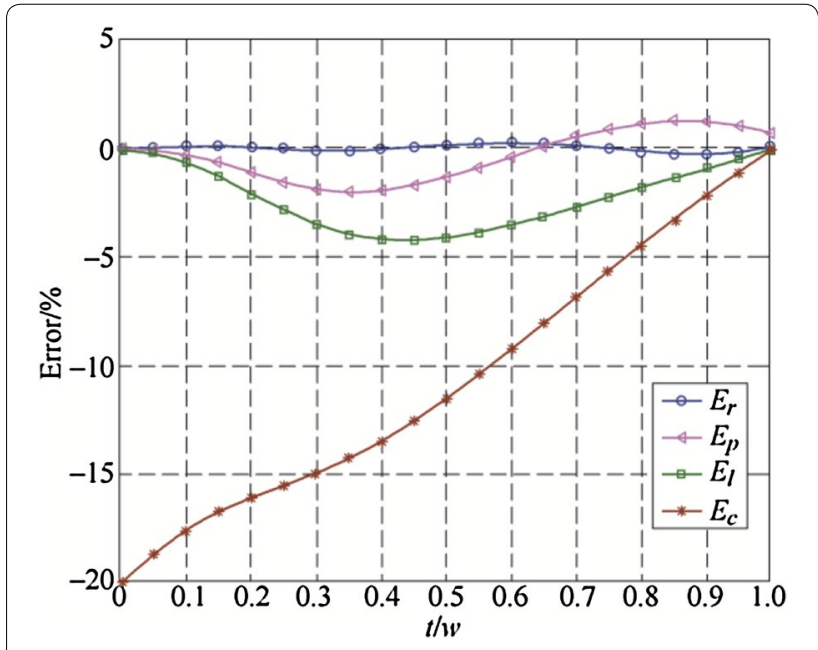

Figure 3 Error comparison of different expressions for Q cross-section must be defined as the wider of the two dimensions because they assume that $w \geq t$. That is to say, if $w$ (width) is no larger than $t$ (thickness), this expression for $\tau_{\max }$ needs to be changed by switching $w$ and $t$. This situation might be troublesome and error-prone during design phase (we always do not know which one is bigger in advance).

\section{General Equations with Symmetric Relation of $t$ and $w$}

A two step procedure was taken to obtain a symmetric and accurate expression for maximum torsion stress $\tau_{\max }$.

First, a symmetric base expression is formulated by revising Eq. (9) as:

$$
Q_{\mathrm{c}}=0.8+0.8 \frac{t}{w},
$$

which yields another equation for $\tau_{\max }$ :

$$
\tau_{\max }=\frac{3 T}{w t^{2}}\left(0.8+0.8 \frac{t}{w}\right) .
$$

As shown in Figure 3, this equation may result in an error up to $20 \%$ (the error is defined as $\left.E_{c}=\left(Q_{c}-Q_{s}\right) / Q_{s}\right)$.

A compensation function, $f(v)$, can be used to reduce the error, where

$$
\tau_{\max }=\frac{2.4 T}{w^{2} t^{2}}(w+t) f(v) .
$$

The variable in the compensation function is chosen to be

$$
v=\left|\log \frac{t}{w}\right|,
$$

because it is symmetric with respect to $t$ and $w$ due to $|\log (\mathrm{t} / \mathrm{w})|=|\log (w / t)|$.

By fitting the results of $E_{c}$ using a quadratic/quadratic rational function, the following compensation function

$$
f(v)=\frac{1.271 v^{2}+0.2829 v+0.0498}{v^{2}+0.27 v+0.0496}
$$

is found to reduce the maximum error to $0.4 \%$ (see Figure 4). This leads to the following expression for $\tau_{\max }$ :

$$
\tau_{\max }=\frac{T(2.4 w+2.4 t)}{w^{2} t^{2}} \frac{1.271 v^{2}+0.2829 v+0.0498}{v^{2}+0.27 v+0.0496} .
$$

Even better fitting results may be achieved with a 4th degree polynomial numerator and 4th degree polynomial denominator:

$$
f^{\prime}(v)=\frac{1.23 v^{4}-0.7167 v^{3}+0.5894 v^{2}-0.2376 v+0.2295}{v^{4}-0.6778 v^{3}+0.6941 v^{2}-0.3427 v+0.2293} .
$$




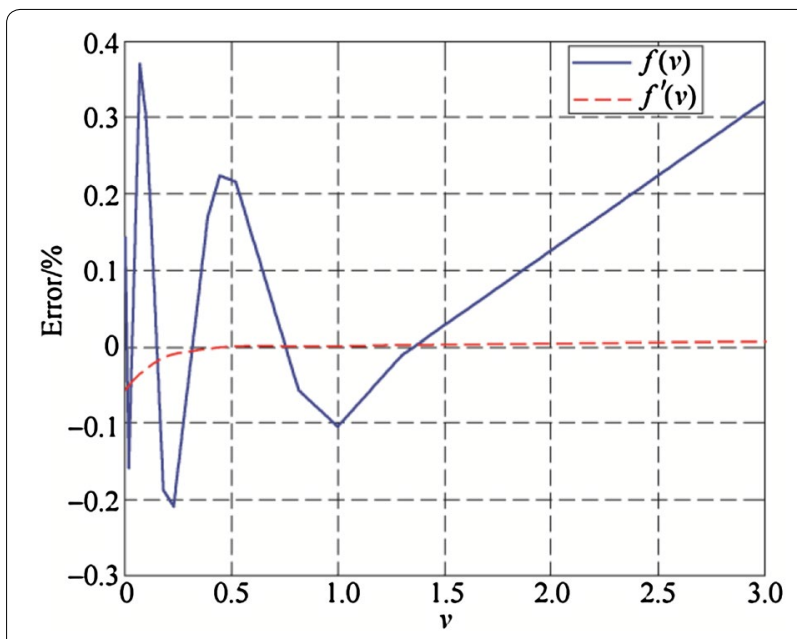

Figure 4 Errors of using compensation function $f(v)$ and $f^{\prime}(v)$

This compensation function reduces the maximum error to $0.07 \%$ (see Figure 4) and leads to the following expression for $\tau_{\text {max }}$ :

$$
\tau_{\max }=\frac{T(w+t)}{w^{2} t^{2}} \frac{2.952 v^{4}-1.72 v^{3}+1.4146 v^{2}-0.5702 v+0.5508}{v^{4}-0.6778 v^{3}+0.6941 v^{2}-0.3427 v+0.2293}
$$

In the following section, we will demonstrate the use of this general (width-thickness independent) equation for designing lamina emergent torsional (LET) joints.

\section{Outside LET Joint: Design Considerations}

Figure 5 shows an outside LET joint fabricated from a planar sheet of material whose modulus of elasticity and modulus of rigidity are $E$ and $G$, respectively. The joint consists of two parallel sets of torsional segments connected by connecting segments in bending. The geometric parameters of the torsional segments are shown in Figure 5 , and $I=w t^{3} / 12$ represents the area moment of inertia in the sheet plane.

\subsection{Torsional Segment: Stiffness vs. Stress}

The stiffness of each torsional segment is expressed using the symmetric equation obtained in Ref. [17] as

$$
K_{t}=\frac{2 G t^{3} w^{3}\left(1.17 \frac{t^{2}}{w^{2}}+2.191 \frac{t}{w}+1.17\right)}{7 L\left(t^{2}+w^{2}\right)\left(\frac{t^{2}}{w^{2}}+2.609 \frac{t}{w}+1\right)}=\frac{G}{L} \cdot k_{c},
$$

where $k_{c}$ is the torsional stiffness constant [23] that is solely determined by the dimensions of the cross-section:

$$
k_{c}=\frac{2 t^{3} w^{3}}{7\left(t^{2}+w^{2}\right)} \cdot \frac{1.17 \frac{t^{2}}{w^{2}}+2.191 \frac{t}{w}+1.17}{\frac{t^{2}}{w^{2}}+2.609 \frac{t}{w}+1} .
$$

As to the maximum stress, we define a stress constant as

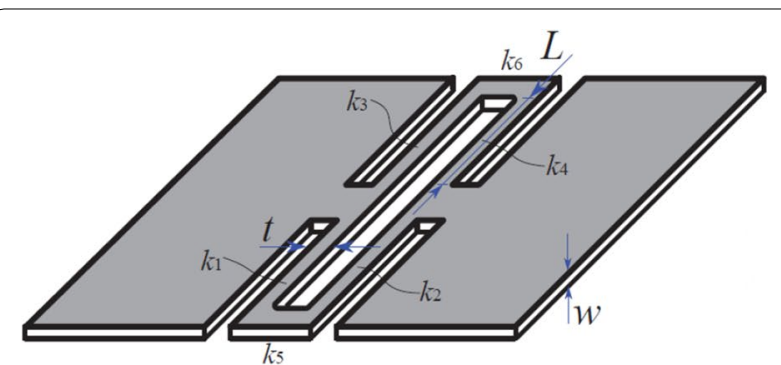

(a)

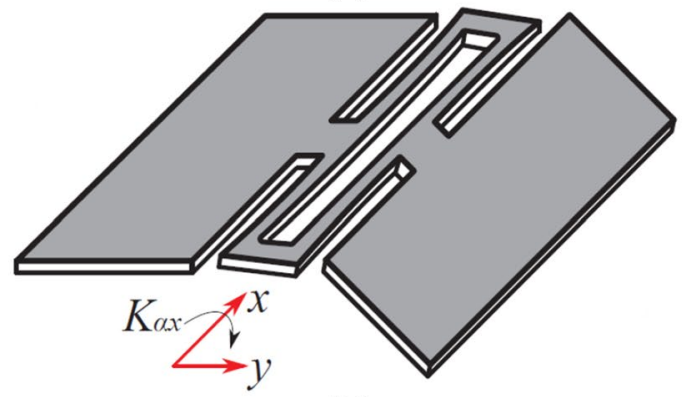

(b)

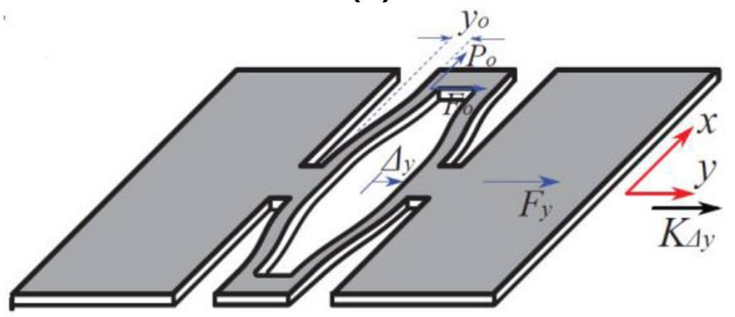

(c)

Figure $\mathbf{5}$ a Outside LET, $\mathbf{b}$ its torsional deflection, and c unconstrained parasitic deflection

$\tau_{c}=\frac{\tau_{\max }}{T}=\frac{2.4 w+2.4 t}{w^{2} t^{2}} \cdot \frac{1.271 v^{2}+0.2829 v+0.0498}{v^{2}+0.27 v+0.0496}$,

which is also solely determined by the dimensions of the cross-section.

If using meters as the length dimensions (e.g., $t$ and $w$ ), the units for $k_{c}$ and $\tau_{c}$ are $\mathrm{m}^{4}$ and $\mathrm{m}^{-3}$, respectively. To compare the twist performance of rectangular cross-sections with different aspect ratios (i.e., $t / w$ ), we define the following non-dimensionalized term called the torsional aspect-ratio factor:

$$
\begin{aligned}
T_{c}=k_{c}^{3} \tau_{c}^{4}= & \frac{0.7738 t w(t+w)^{4}}{\left(t^{2}+w^{2}\right)^{3}} . \\
& \frac{\left(1.17 \frac{t^{2}}{w^{2}}+2.191 \frac{t}{w}+1.17\right)^{3}}{\left(\frac{t^{2}}{w^{2}}+2.609 \frac{t}{w}+1\right)^{3}} . \\
& \frac{\left(1.271 v^{2}+0.2829 v+0.0498\right)^{4}}{\left(v^{2}+0.27 v+0.0496\right)^{4}},
\end{aligned}
$$


$T_{c}$ is also symmetric with respect to $t$ and $w$ because it is only determined by the width-thickness ratio of the cross-section. Figure 6 plots $T_{c}$ as a function of $t / w$. For a LET joint design, we always expect $k_{c}$ to be small so as to lower the torsional stiffness in the desired direction for reducing actuation effort, and $\tau_{c}$ to be small to increase the maximum allowed rotation angle. $T_{c}$ reaches its maximum, 2.2747, at $t / w=0.658$ and $t / w=1.52 . T_{c}$ reaches its local minimum, 1.4942, at $t / w=1$ (square cross-section). $T_{c}$ is also smaller than 1.4942 for $t / w<0.35$ and $t / w$ $>2.86$. In general, cross-sections of $0.35<t / w<1$ and 1 $<t / w<2.86$ are suggested to be avoided for the torsional segments in LET joints.

It is obvious that increasing the length can significantly decrease $F$, which is preferred if the space is allowed. If $L$ is fixed, there is a local minimum at $t / w=1$. However, there are two maxima at $t / w=1.5$ and $t / w=0.67$ (these geometries are feasible and preferred both for design and manufacture), which are suggested to be avoided in design.

\subsection{LET Joint: Torsional Stiffness vs. Compressive/Tensile Stiffness}

Ideally a LET joint would have low torsional stiffness while maintaining high stiffness in the other directions [10]. However, a LET joint is susceptible to undesired motion when compressive/tensile load is applied because the torsional segments are placed into bending, as illustrated in Figure 5(c).

When an outside LET joint is subject to a compressive/tensile load, the torsional segments can be treated as fixed-guided segments with the axial force $P_{o}=0$ (because the connecting segments is floating) as illustrated in Figure 5. The transverse force $F_{o}$, can be

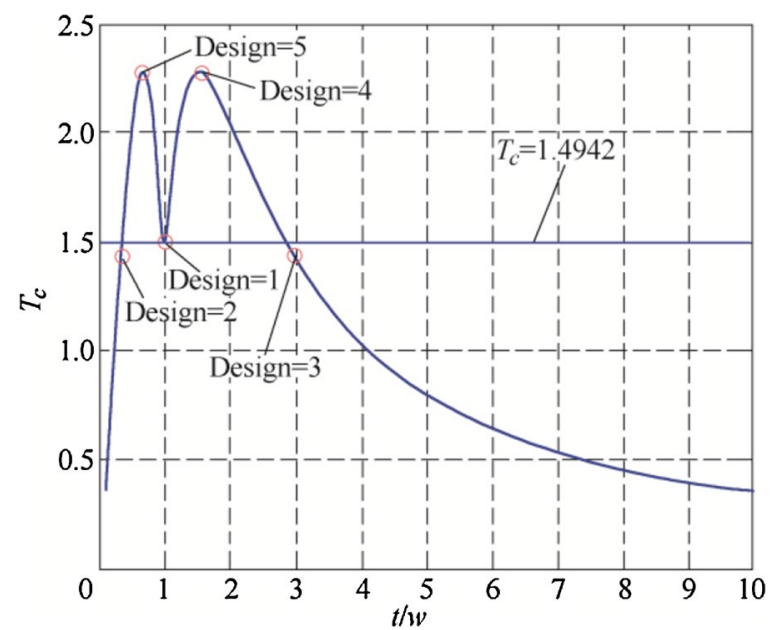

Figure $6 T_{c} v s . t / W$ expressed by the Bi-BCM (the first bending mode) [27] as:

$$
f_{o}=\frac{9 p_{o}^{2}+312 p_{o}+720}{16 p_{o}+480} y_{o}=\frac{3 y_{o}}{2},
$$

in which $f_{o}$ and $p_{o}$ are the normalized axial and transverse forces, respectively, defined as

$$
p_{o}=\frac{P_{o} L^{2}}{4 E I}=0, \quad f_{o}=\frac{F_{o} L^{2}}{4 E I},
$$

and $y_{o}$ is the normalized displacement defined as

$$
y_{o}=\frac{2 Y_{o}}{L} \text {. }
$$

By substituting Eqs. (27) and (28) into Eq. (26), the parasitic compression/extension stiffness of the whole LET joint can be obtained as:

$$
K_{\Delta y}=\frac{F_{y}}{\Delta_{y}}=\frac{2 F_{o}}{2 Y_{o}}=\frac{12 E I}{L^{3}}=\frac{E w t^{3}}{L^{3}} .
$$

The equivalent torsional compliance of the outside LET joint along the $x$-axis [10] is:

$$
K_{\alpha x}=\frac{k_{1} k_{2} k_{5}}{k_{1} k_{2}+k_{1} k_{5}+k_{2} k_{5}}+\frac{k_{3} k_{4} k_{6}}{k_{3} k_{4}+k_{3} k_{6}+k_{4} k_{6}},
$$

where the stiffnesses of the torsional segments are

$$
k_{1}=k_{2}=k_{3}=k_{4}=K_{t},
$$

while the connecting segments in bending can be considered stiff because they are short, i.e.,

$$
k_{5}=k_{6}=\infty \text {. }
$$

Then we have

$$
K_{\alpha x}=\frac{2 G t^{3} w^{3}\left(1.17 \frac{t^{2}}{w^{2}}+2.191 \frac{t}{w}+1.17\right)}{7 L\left(t^{2}+w^{2}\right)\left(\frac{t^{2}}{w^{2}}+2.609 \frac{t}{w}+1\right)} .
$$

For the purpose of comparing the compression/extension stiffness to the torsional stiffness of LET joints for different design parameters, the following stiffness ratio term is defined:

$$
\begin{aligned}
R_{s}= & \frac{K_{\Delta y}}{K_{\alpha x}}=\frac{7}{2} \cdot \frac{E}{G} \cdot \frac{1}{L^{2}} . \\
& \frac{\frac{t^{4}}{w^{4}}+2.609 \frac{t^{3}}{w^{3}}+2 \frac{t^{2}}{w^{2}}+2.609 \frac{t}{w}+1}{1.17 \frac{t^{2}}{w^{2}}+2.191 \frac{t}{w}+1.17} .
\end{aligned}
$$

A well-designed LET joint should have large $R_{s}$ so as to achieve low torsional stiffness but provide good constraint in the compression/extension direction. The right 
side of Eq. (34) is divided into four terms, among which the first is a constant, the second is material-related term, the third is inversely proportional to the square of the length of the torsional segments, while the last monotonically increases with $t / w$. For isotropic materials, the Poisson's ratio $v$ ranges from 0.2 to 0.5 [28], thus the range of the second term $E / G=2(1+v)$ is from 2.4 to 3 . However, one can use composite materials to obtain larger $E / G$ (e.g., multi-layer structures). The third term indicates that $R_{s}$ can be significantly increased by decreasing the length of the torsional segments. However, decreasing the length of the torsional segments will increase the torsional stiffness (as indicated by Eq. (22)) and further decrease rotational range of the joint. The fourth term is plotted in Figure 7 as a function of $w / t$, which shows that $R_{s}$ dramatically increases as $w / t$ increases. Considering $T_{c}$ shown in Figure $6, w / t>2.86$ is suggested if parasitic motion along the compression/extension direction is required to be small.

\subsection{Design Examples}

This section provides a few LET joint designs to demonstrate the design considerations in the previous section. The parameters of 5 designs are listed in Table 1 . These

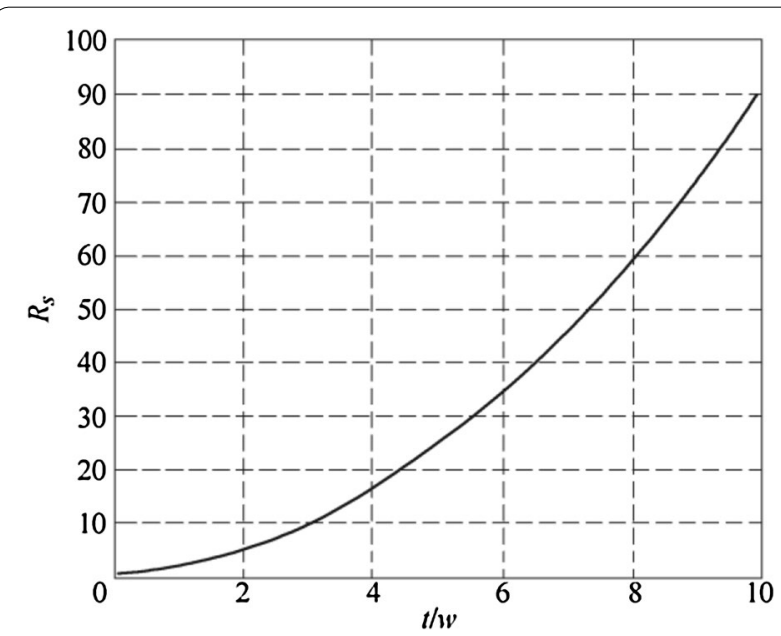

Figure $7 R_{s}$ as a function of $t / w$ designs have equal or almost equal cross-section area. Figure 6 marks these designs on the $T_{c}$ curve.

\section{Considering}

$$
T=K_{\alpha x} \alpha,
$$

where $\alpha$ is the torsional deflection angle of the joint, substituting it into Eq. (19) yield

$$
\begin{aligned}
\tau_{\max }= & \frac{0.689 G}{L} \cdot \frac{t w(w+t)}{w^{2}+t^{2}} \cdot \frac{1.271 v^{2}+0.2829 v+0.0498}{v^{2}+0.27 v+0.0496} . \\
& \frac{1.17 \frac{t^{2}}{w^{2}}+2.191 \frac{t}{w}+1.17}{\frac{t^{2}}{w^{2}}+2.609 \frac{t}{w}+1} \cdot \alpha .
\end{aligned}
$$

For the purpose of comparison, we calculated $\tau_{\max }$ using Eq. (36) by assuming $\alpha=0.1 \mathrm{rad}$.

The results of $K_{\alpha x}, K_{\Delta y}$ and $\tau_{\max }$ for the 5 designs are listed in Table 2. Among the 5 joints, Design 3 is the best design, with the smallest $K_{\alpha x}$, the largest $K_{\Delta y}$, and the lowest $\tau_{\max }$. Design 2 has the same values for $K_{\alpha x}$ and $\tau_{\max }$ as Design 3, but has the lowest compression/extension stiffness. Design 1 has modest values for $K_{\Delta y}$ and $\tau_{\max }$ but the largest $K_{\alpha x}$. Design 4 and Design 5 are the worst designs because they have the largest $K_{\alpha x}$ and $\tau_{\max }$.

\section{Conclusions}

This work presented closed-form symmetric equations for calculating maximum torsion stress of a rectangular cross-section beam. Together with the symmetric equations in our previous work [17], these equations are independent of the relative magnitude of the two dimensions (i.e., the thickness and the width) of the cross-section, thus are more convenient and effective for designing and optimizing torsional beams in compliant mechanisms.

Table 2 Calculated results

\begin{tabular}{|c|c|c|c|c|c|c|}
\hline & $t(\mathrm{~mm})$ & $w(\mathrm{~mm})$ & $t / w$ & $L(\mathrm{~mm})$ & $E(\mathrm{GPa})$ & $\overline{G(\mathrm{GPa})}$ \\
\hline Design 1 & 2 & 2 & 1 & 10 & 205 & 79.37 \\
\hline Design 2 & 1.16 & 3.45 & 0.336 & 10 & 205 & 79.37 \\
\hline Design 3 & 3.45 & 1.16 & 2.97 & 10 & 205 & 79.37 \\
\hline Design 4 & 2.5 & 1.6 & 1.56 & 10 & 205 & 79.37 \\
\hline Design 5 & 1.6 & 2.5 & 0.64 & 10 & 205 & 79.37 \\
\hline
\end{tabular}

\begin{tabular}{llll}
\hline & $\boldsymbol{K}_{\boldsymbol{\alpha} \boldsymbol{x}}(\mathbf{N} \cdot \mathbf{m})$ & $\boldsymbol{K}_{\boldsymbol{\Delta} \boldsymbol{y}}(\mathbf{N} / \mathbf{m})$ & $\boldsymbol{\tau}_{\max }$ \\
\hline Design 1 & 17.8347 & 3280000 & 1 \\
Design 2 & 11.2398 & 1103900 & 0.336 \\
Design 3 & 11.2398 & 9764900 & 2.97 \\
Design 4 & 16.3246 & 5125000 & 1.56 \\
Design 5 & 16.3246 & 2099200 & 0.64 \\
\hline
\end{tabular}

Table 1 A few LET joint designs 
These equations were utilized to analyze outside lamina emergent torsional joints and some useful design insights were obtained and described.

\section{Authors' contributions}

GC and LLH conceived the idea, GC carried out the calculation, and GC and LLH drafted the manuscript. All authors read and approved the final manuscript.

\section{Author details}

'State Key Laboratory for Manufacturing Systems Engineering, Xi'an Jiaotong University, Xi'an 710049, Shaanxi, China. ${ }^{2}$ Department of Mechanical Engineering, Brigham Young University, Provo, UT 84602, USA.

\begin{abstract}
Authors' Information
Gui-Min Chen is a professor at School of Mechanical Engineering, Xi'an Jiaotong University, China. He received his PhD, MS and BS degrees from Xidian University, China. He was a visiting professor at Brigham Young University, US. His research interests include compliant mechanisms and their applications. He serves as an associate editor for the Journal of Mechanisms and Robotics.

Larry L. Howell is a professor and associate dean of the Department of Mechanical Engineering, Brigham Young University, US, where he also holds a University Professorship. Prior to joining BYU in 1994 he was a visiting professor at Purdue University, a finite element analysis consultant for Engineering Methods, and an engineer on the design of the YF-22 (the prototype for the U.S. Air Force F-22). He received his PhD and MS degrees from Purdue University and his BS from Brigham Young University. He is a licensed professional engineer and the recipient of a National Science Foundation CAREER Award, a Theodore von Kármán Fellowship, the BYU Technology Transfer Award, the Maeser Research Award, several best paper awards, and the ASME Mechanisms \& Robotics Award. He is a Fellow of ASME, associate editor for the Journal of Mechanisms and Robotics, past chair of the ASME Mechanisms \& Robotics Committee, past co-chair of the ASME International Design Engineering Technical Conferences, and a past Associate Editor for the Journal of Mechanical Design. Prof. Howell's technical publications and patents focus on compliant mechanisms, including origami-inspired mechanisms, microelectromechanical systems, and medical devices. He is the author of the book Compliant Mechanisms published by John Wiley \& Sons.
\end{abstract}

\section{Acknowledgements}

Supported by National Science Foundation Research of the United States (Grant No. 1663345), National Natural Science Foundation of China (Grant No. 51675396), and Fundamental Research Fund for the Central Universities (Grant No. 12K5051204021).

\section{Competing interests}

The authors declare that they have no competing interests.

\section{Ethics approval and consent to participate}

Not applicable.

\section{Publisher's Note}

Springer Nature remains neutral with regard to jurisdictional claims in published maps and institutional affiliations.

Received: 31 May 2017 Accepted: 14 January 2018

Published online: 27 February 2018

\section{References}

1. L L Howell. Compliant mechanisms. New York: Wiley, 2001

2. N Wang, L X iang, X Zhang. Pseudo-rigid-body Model for Corrugated Cantilever Beam Used in Compliant Mechanisms. Chinese Journal of Mechanical Engineering, 2014, 27(1): 122-129.

3. U Hanke, P Hampel, A Comsa, et al. Compliant mechanism synthesis by using elastic similitude. Chinese Journal of Mechanical Engineering, 2015, 28(4): 769-775.
4. S Liu, J Dai, A Li, et al. Analysis of frequency characteristics and sensitivity of compliant mechanisms. Chinese Journal of Mechanical Engineering, 2016, 29(4): 680-693.

5. M Goldfarb, J E Speich. A well-behaved revolute flexure joint for compliant mechanism design. ASME Journal of Mechanical Design, 1999, 121 : 424-429.

6. B P Trease, Y.-M. Moon, S Kota. Design of large-displacement compliant joints. ASME Journal of Mechanical Design, 2005, 127(7): 788-798.

7. O Degani, E Socher, A Lipson, et al. Pull-in study of an electrostratic torsion microactuator. Journal of Microelectromechanical Systems, 1998, 7: $373-379$

8. M Bao, Y Sun, J Zhou, et al. Squeeze-film air damping of a torsion mirror at a finite tilting angle. Journal of Micromechanics and Microengineering, 2006, 16: 2330-2335.

9. N Lobontiu, B Ilic, E Garcia, et al. Modeling of nanofabricated paddle bridges for resonant mass sensing. Review of Scientific Instruments, 2006 77: 073301.

10. J O Jacobsen, $G$ Chen, L L Howell, et al. Lamina emergent torsion (LET) joint. Mechanism and Machine Theory, 2009, 44(11): 2098-2109.

11. Z Xie, L Qiu, D Yang. Design and analysis of Outside-Deployed Lamina Emergent Joint (OD-LEJ). Mechanism and Machine Theory, 2017, 114: $111-124$

12. S E Wilding, L L Howell, S P Magleby. Introduction of planar compliant joints designed for compressive and tensile loading conditions in Lamina Emergent Mechanisms. Mechanism and Machine Theory, 2012, 56: 1-15.

13. K J Boehm, C R Gibson, J R Hollaway, et al. A flexure-based mechanism for precision adjustment of national ignition facility target shrouds in three rotational degrees of freedom. Fusion Science and Technology, 2016, 70(2): 265-273.

14. T G Nelson, R L Lang, N Pehrson, et al. Facilitating deployable mechanisms and structures via developable Lamina emergent arrays. ASME Journal of Mechanisms and Robotics, 2016, 8: 031006.

15. I L Delimont, S P Magleby, L L Howell. Evaluating compliant hinge geometries for origami-inspired mechanisms. ASME Journal of Mechanisms and Robotics, 2015, 7(1): 011009

16. G Radaellia, R Buskermolenb, R Barentsc, et al. Static balancing of an inverted pendulum with prestressed torsion bars. Mechanism and Machine Theory, 2017, 108: 14-26.

17. $\mathrm{G}$ Chen, $\mathrm{L} L$ Howell. Two general solutions of torsional compliance for variable rectangular cross-section hinges in compliant mechanisms. Precis. Eng., 2009, 33: 268-274

18. Z Zhu, X Zhou, R Wang, et al. A simple compliance modeling method for flexure hinges. Science China Technological Sciences, 2015, 58(1): 56-63.

19. Z Zhu, S To. Characterization of spatial parasitic motions of compliant mechanisms induced by manufacturing errors. ASME Journal of Mechanisms and Robotics, 2016, 8(1): 011018.

20. Y Shen, X Chen, W Jiang, et al. Spatial force-based non-prismatic beam element for static and dynamic analysis of circular flexure hinges in compliant mechanisms. Precision Engineering, 2014, 38(2): 311-320.

21. G Chen, R Bai. Modeling large spatial deflections of slender bisymmetric beams in compliant mechanisms using chained spatial-beam-constraintmodel. ASME Journal of Mechanisms and Robotics, 2016, 8(4): 041011.

22. O A Turkkan, H Su. Towards computer aided design and analysis of spatia flexure mechanisms. ASME 2016 International Design Engineering Technical Conferences and Computers and Information in Engineering Conference, Charlotte, North Carolina, USA, August 21-24, 2016, DETC2016-59966.

23. S P Timoshenko, J N Goodier. Theory of elasticity. McGraw-Hill, 1970.

24. W C Young, R G Budynas. Roark's formulas for stress and strain. John Wiley \& Sons, 2005

25. W D Pilkey. Formulas for stress, strain, and structural matrices (2nd ed.). McGraw Hill, 2002.

26. R J Roark. Formulas for stress and strain (5nd ed.). New York: McGraw Hill, 1975.

27. F Ma, G Chen. Bi-BCM: A closed-form solution for fixed-guided beams in compliant mechanisms. ASME Journal of Mechanisms and Robotics, 2017,9, 1, 014501.

28. G N Greaves, A L Greer, R S Lakes, et al. Poisson's ratio and modern materials. Nature Materials, 2011, 10:823-837. 